\title{
Non-Clinical Pharmacokinetic/Pharmacodynamic and Early Clinical Studies Supporting Development of a Novel Subcutaneous Formulation for the Monoclonal Antibody Rituximab
}

\author{
Authors \\ Affiliations \\ B. Bittner ${ }^{1,2}$, W. F. Richter ${ }^{3}$, F. Hourcade-Potelleret ${ }^{4}$, F. Herting ${ }^{5}$, J. Schmidt \\ F. Hoffmann-La Roche Ltd., Product Optimization, Basel, Switzerland \\ F. Hoffmann-La Roche Ltd., Clinical Pharmacology, Basel, Switzerland \\ ${ }^{3}$ F. Hoffmann-La Roche Ltd., Non-clinical Safety, Basel, Switzerland \\ ${ }^{4}$ F. Hoffmann-La Roche Ltd., Clinical Modeling \& Simulation, Basel, Switzerland \\ ${ }^{5}$ Roche Diagnostics GmbH, Penzberg, Germany
}

Key words

clinical trials

dose finding

- follicular lymphoma

- intravenous

pharmacology

- preclinical studies

received 24.07.2013

accepted 26.12.2013

Bibliography

DOI http://dx.doi.org/

10.1055/s-0033-1363993

Published online:

January 22, 2014

Drug Res 2014;

64: 569-575

(c) Georg Thieme Verlag KG

Stuttgart - New York

ISSN 2194-9379

Correspondence

J. Schmidt

Product Optimization

F. Hoffmann-La Roche Ltd.

Grenzacher Strasse 124

$\mathrm{CH}-4070$, Basel

Switzerland

Tel.: +41/61/688 2120

Fax: $+41 / 61 / 6880977$

johannes.schmidt@roche.com

\section{Abstract \\ $\nabla$}

This overview article describes the non-clinical pharmacology, pharmacokinetic and clinical dose-finding programs supporting the development of a novel subcutaneous formulation for rituximab, a monoclonal antibody that selectively targets CD20-positive B-lymphocytes. The subcutaneous route of administration is expected to improve convenience for patients and to reduce healthcare professional resource use compared with conventional intravenous infusion. Various non-clinical and clinical studies were conducted to support the bridge from the approved intravenous formulation to the novel subcutaneous treatment. The underlying hypothesis for these studies was that achieving subcutaneous rituximab serum trough concen- trations that are at least as high as those reached with the intravenous formulation would result in at least the same degree of receptor saturation. Preclinical mouse xenograft and cynomolgus monkey B-cell depletion studies were performed at intravenous and subcutaneous doses that were previously found to result in comparable serum concentrations in pharmacokinetic studies in the same species. Results from these non-clinical assessments guided dose selection for the subsequent phase $1 \mathrm{~b}$ dose finding trials in patients with follicular lymphoma as part of maintenance treatment. A fixed dose of $1400 \mathrm{mg}$ was found to result in noninferior serum trough concentrations to the intravenous formulation. Clinical trials in the induction setting in patients with follicular lymphoma and chronic lymphocytic leukemia are currently ongoing.

\begin{tabular}{|c|c|c|c|}
\hline \multirow{2}{*}{\multicolumn{2}{|c|}{$\begin{array}{l}\text { Abbreviations } \\
\nabla\end{array}$}} & $\mathrm{mAb}$ & monoclonal antibody \\
\hline & & ORR & overall response rate \\
\hline \multirow[t]{2}{*}{ AUC } & area under the serum concentration- & PK & pharmacokinetic \\
\hline & time curve & PR & partial response \\
\hline BSA & body surface area & $\mathrm{R}-\mathrm{CHOP}$ & rituximab plus cyclophosphamide, \\
\hline CD20+ & CD20-positive & & doxorubicin, vincristine and \\
\hline CHOP & cyclophosphamide, doxorubicin, & & prednisone \\
\hline & vincristine and prednisone & rHuPH20 & recombinant human hyaluronidase \\
\hline $\mathrm{CI}$ & confidence interval & SC & subcutaneous \\
\hline CLL & chronic lymphocytic leukemia & SD & standard deviation \\
\hline CR & complete response & $\mathrm{T}_{\max }$ & time to maximum serum concentra- \\
\hline $\mathrm{CRu}$ & unconfirmed complete response & & tion \\
\hline $\mathrm{C}_{\max }$ & maximum concentration & $\mathrm{U}$ & units \\
\hline
\end{tabular}

\section{Introduction}

Rituximab is a chimeric murine/human monoclonal antibody $(\mathrm{mAb})$ that specifically binds to CD20, a hydrophobic trans-membrane protein present on the surface of B-lymphocytes [1]. Over the past decade, rituximab has become the standard of care for management of patients 
suffering from various B-cell malignancies, including follicular lymphomas (FL), diffuse large B-cell lymphoma (DLBCL) and chronic lymphocytic leukemia (CLL) [2].

Rituximab is administered as an intravenous (IV) infusion over several hours and frequently, infusion-related reactions may require further prolongation of the infusion time [3]. The procedure required to establish IV access is considered invasive and can be painful, particularly in patients with malignant diseases who are treated repeatedly [4]. To overcome these issues, a subcutaneous (SC) formulation of rituximab is currently in development. Subcutaneous administration of rituximab takes less than $10 \mathrm{~min}$ and is a simple alternative to the current practice of IV administration. This new route of administration could thus reduce the time a patient spends in the hospital and eliminate hospital burden associated with IV administration (e.g., preparation of the infusion bag, nursing time for IV dosing, rental of day beds) as has been reported for the SC Vs. IV administration of heparin or for the mAb trastuzumab $[5,6]$.

Data from a multicenter, prospective, time-and-motion study of trastuzumab (PrefHer) showed that, compared with IV infusion, active healthcare professional time for the SC injection using a single-use injection device was reduced by $28 \%$ in Denmark and $34 \%$ in France [6]. In addition, patient chair time was reduced by $68-69 \%$ across both countries. These improvements could ultimately result in an increase in the number of patients receiving treatment with trastuzumab, especially in countries that have limited infusion capacity. Furthermore, both patients and healthcare professionals prefer the SC route of administration; in the PrefHer study, $91.5 \%$ of 236 patients and $73.8 \%$ of 103 healthcare professionals preferred the SC formulation to IV administration [6].

This review article describes the non-clinical pharmacology/ pharmacokinetics (PKs) and early clinical approach supporting the development of a novel SC formulation of rituximab. Early development activities for a novel SC formulation for the $\mathrm{mAb}$ trastuzumab have been described previously [7], and will be referred to as appropriate.

\section{Scientific Basis for Dose Selection Approach}

In line with the dose selection approach for the SC formulation of trastuzumab [7], the aim of the dose-finding studies reviewed here was to select a dose of rituximab SC that achieves serum trough concentrations $\left(\mathrm{C}_{\text {trough }}\right)$ at least as high as those reached with the approved IV formulation, while maintaining the clinical dosing frequency for a given indication. The selected SC dose was then to be confirmed by a formal PK noninferiority test to compare it against the approved IV regimen. The key assumption supporting this dose selection approach was that clinical response is driven by the concentration of a given $\mathrm{mAb}$, and that optimal efficacy is obtained when all accessible target sites are saturated.

The validity of this PK-based bridging concept is supported by the clinical data generated for the SC formulation of trastuzumab. As part of the trastuzumab clinical program, a phase I study was conducted in healthy male volunteers and female patients with HER2-positive early breast cancer. This study was designed to select a fixed SC dose of trastuzumab that resulted in $\mathrm{C}_{\text {trough }}$ and area under the serum concentration-time curve (AUC) values at least as high as those achieved with the approved IV regimen [8]. The selected fixed dose of $600 \mathrm{mg}$ was subsequently confirmed in a phase III study, which showed that noninferior trastuzumab serum trough concentrations achieved using SC dosing result in comparable efficacy (measured as pathologic complete response) to the IV regimen [9].

\section{Approved Rituximab Doses and Dosing Regimens} $\nabla$

The recommended dose of rituximab for adult patients in the approved non-Hodgkin's lymphoma indications is $375 \mathrm{mg} / \mathrm{m}^{2}$ body surface area (BSA) per IV infusion [10]. 4 different dosing schedules are currently approved ( $\bullet$ Table 1 ). The recommended dose of rituximab for previously untreated and relapsed/refractory patients with CLL is $375 \mathrm{mg} / \mathrm{m}^{2} \mathrm{BSA}$ administered on day 0 of the first treatment cycle, followed by $500 \mathrm{mg} / \mathrm{m}^{2}$ BSA administered on day 1 of each subsequent cycle for 6 cycles in total [10].

\section{Rituximab SC Formulation \\ $\nabla$}

Intravenous MabThera ${ }^{\circledR}$ (rituximab) is approved as a liquid formulation containing $10 \mathrm{mg} / \mathrm{mL}$ rituximab (excipients: sodium citrate, polysorbate 80 , sodium chloride, sodium hydroxide, hydrochloric acid and water for injections) that is available in 2 vial sizes containing $10 \mathrm{~mL}$ and $50 \mathrm{~mL}$ of MabThera ${ }^{\mathrm{TM}}$, respectively.

The development of a ready-to-use SC formulation of rituximab became feasible with the recent advances in the preparation of highly concentrated $\mathrm{mAb}$ formulations $[11,12]$ and the availability of recombinant human hyaluronidase (rHuPH20), an enzyme that enables rapid SC administration of volumes exceeding $2 \mathrm{~mL}$ [13]. A liquid formulation of MabThera containing

Table 1 European Medicines Agency approved rituximab dosing regimens [10].

\begin{tabular}{|ll} 
Regimen & $\begin{array}{l}\text { Indication } \\
\text { Monotherapy }\end{array}$ \\
\hline $\begin{array}{l}\text { Combination therapy } \\
\text { b }\end{array}$ & Relapsed/refractory FL (stage 3-4) \\
\hline R-chemotherapy & $\begin{array}{l}\text { Previously untreated or relapsed/refractory } \\
\text { (stage 3-4) FL }\end{array}$ \\
\hline
\end{tabular}

Previously untreated or relapsed/refractory CLL

R-CHOP

DLBCL

\section{Frequency}

Induction or retreatment: q1w

Induction: day 1 of each chemotherapy cycle

Maintenance: q2m for untreated FL; q3m for relapsed/refractory $\mathrm{FL}$

Day 0 of first treatment cycle then day 1 of each subsequent cycle ${ }^{c}$ Day 1 of each chemotherapy cycle

Treatment duration
4 weeks
8 cycles
Until PD or up to 2 years
6 cycles
8 cycles

a Patients who respond to rituximab induction treatment can be treated again with rituximab. ${ }^{\mathrm{b}}$ Rituximab should be administered on day 1 of each chemotherapy cycle after IV administration of the glucocorticoid component of the chemotherapy, if applicable. ${ }^{\mathrm{c}}$ First dose $375 \mathrm{mg} / \mathrm{m}^{2}$, followed by $500 \mathrm{mg} / \mathrm{m}^{2}$ for cycles $2-6$. PD: disease progression; q1w: weekly; q2m: every 2 months; q3m: every 3 months; R-CHOP: rituximab plus CHOP 


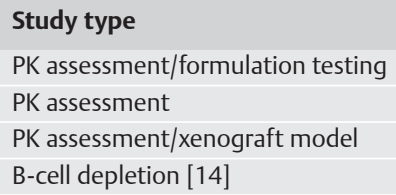

\begin{tabular}{ll} 
Species and strain & Route of administration \\
Minipig/Göttingen & IV and SC \\
Cynomolgus monkey & SC \\
\hline Mouse/SCID beige mice & IV and SC \\
Cynomolgus monkey & IV and SC
\end{tabular}

Table 2 Overview of non-clinical PK and pharmacodynamic studies for rituximab SC.

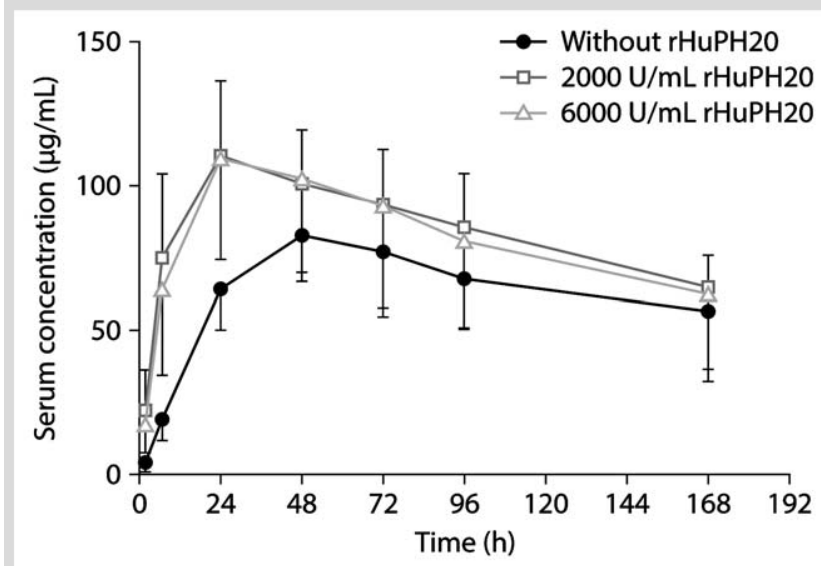

Fig. 1 Time course of rituximab serum concentrations in minipigs following single subcutaneous administration of rituximab at $120 \mathrm{mg} /$ animal containing either 0,2000 or $6000 \mathrm{U} / \mathrm{mL}$ rHuPH20 ( $n=5$ per dose group).

$120 \mathrm{mg} / \mathrm{mL}$ rituximab and 2000 units (U)/mL rHuPH20, has been developed for SC administration [14]. This formulation is administered using a syringe with a hypodermic needle.

\section{Non-Clinical Pharmacology and PK Studies Supporting Development of the SC Formulation for Rituximab \\ $\nabla$}

Non-clinical pharmacology and PK studies were conducted to support the design of the clinical dose-finding study by assessing (i) the absorption and elimination of rituximab, and (ii) its preclinical efficacy at similar serum concentrations for the SC and IV administration routes ( $\bullet$ Table 2 ).

\section{Non-Clinical PK Studies}

To explore the effect of different rHuPH20 concentrations on the SC absorption of rituximab, a PK study was conducted in minipigs. This study was similar in design to one carried out during the development of the SC formulation of trastuzumab [7]. 4 groups of female minipigs ( 5 per dose group) received a single IV dose of rituximab at $9.6 \mathrm{mg} / \mathrm{kg}$ or a single SC dose of rituximab at $120 \mathrm{mg} /$ animal (about $14 \mathrm{mg} / \mathrm{kg}$ ) containing 0,2000 or $6000 \mathrm{U} / \mathrm{mL}$ rHuPH20, respectively (for IV and SC dosing of the formulation without rHuPH20 see [15], for other dose groups; data on file, F. Hoffmann-La Roche Ltd.). A fifth group received a dose of rituximab SC at $240 \mathrm{mg} /$ animal $(\approx 27.6 \mathrm{mg} / \mathrm{kg}$ ) containing $2000 \mathrm{U} / \mathrm{mL}$ rHUPH20 to explore dose linearity of SC absorption. The SC injection volumes were 1 and $2 \mathrm{~mL}$ for the $120 \mathrm{mg}$ and $240 \mathrm{mg}$ dose groups, respectively.

The lower trastuzumab SC dose $(120 \mathrm{mg})$ was selected to limit the dose volume to $1 \mathrm{~mL}$, the typical dose volume for conventional SC formulations. The IV dose of $9.6 \mathrm{mg} / \mathrm{kg}$ was selected to obtain a similar systemic exposure to that achieved with the $120 \mathrm{mg} / \mathrm{animal}$ SC dose (about $14 \mathrm{mg} / \mathrm{kg}$ ), assuming a SC bioavailability of approximately $70 \%$.

The rHuPH20 concentrations selected for testing in the minipig model took into consideration results from a clinical study using rHuPH20 co-formulated with an undisclosed large protein molecule therapeutic (LPMT) in rheumatoid arthritis patients [16]. Data suggested a concentration-dependent effect of rHuPH20 on LPMT absorption, with the highest effect on LPMT absorption observed at rHuPH20 concentrations of 1800 and $3500 \mathrm{U} / \mathrm{mL}$. Based on these data, $2 \mathrm{rHuPH} 20$ concentrations ranging from the lower end of maximum effect concentration range $(2000 \mathrm{U} / \mathrm{mL})$ to the highest concentration tested in the LPMT study $(6000 \mathrm{U} /$ $\mathrm{mL}$ ) were selected for the minipig study in order to further assess whether a lower or higher rHuPH20 concentration would result in different absorption kinetics of rituximab. This dose selection was also consistent with results from a mouse dye dispersion study, which demonstrated that administration of a co-formulation containing $500-5000 \mathrm{U} / \mathrm{mL}$ rHuPH20 achieved a dispersion profile similar to sequential administration of a solution containing $100 \mathrm{U} / \mathrm{mL} \mathrm{rHuPH} 20$ followed by dye injection [7].

PK profiles and parameters are shown in $\odot$ Fig. 1 and $\odot$ Table 3. Following SC administration, rituximab absorption was more rapid with rHuPH20-containing formulations. At the SC dose level of $120 \mathrm{mg} / \mathrm{animal}$ the median time to maximum serum levels $\left(T_{\max }\right)$ was shortened from $48 \mathrm{~h}$ without rHuPH20 to $24 \mathrm{~h}$ for both of the rHuPH20-containing formulations. Average maximum serum levels of rituximab $\left(C_{\max }\right)$ for the rHuPH20-containing formulations were increased relative to the levels obtained for the formulation without rHuPH20. The extent of bioavailability (mean \pm standard deviation [SD]) of rituximab SC at $120 \mathrm{mg} /$ animal from non-compartmental PK analysis ranged between 52 and $71 \%$ (data on file, F. Hoffmann-La Roche Ltd.).

Doubling the rituximab SC dose had no obvious impact on absorption and disposition, as shown in $\odot$ Table 3. At the $240 \mathrm{mg}$ dose of rituximab SC, exposure $\left(\mathrm{AUC}_{0-672 \mathrm{~h}}\right.$ and $\mathrm{C}_{\max }$ ) was roughly twice as high as that for the $120 \mathrm{mg}$ SC dose.

Compartmental PK modeling revealed approximately 3-fold higher estimates for the absorption rate constants associated with the rHuPH20-containing formulations relative to the rate constant associated with the control formulation (without rHuPH20). This modeling approach indicated that the average absorption rate constants were $0.0221,0.0803$, and $0.0557 \mathrm{~h}^{-1}$ at 0,2000 and $6000 \mathrm{U} / \mathrm{mL}$ of rHUPH20, respectively. Thus, there was no further increase in the absorption rate constants when increasing the rHuPH20 concentration from 2000 to $6000 \mathrm{U} / \mathrm{mL}$. There was no relevant difference in the absorbed fraction of rituximab from the various formulations. The estimate for the absorbed fraction of rituximab was $68.9 \%$ when utilizing data from all 4 SC dose groups.

A further study was conducted to support planning and SC dose selection for a pharmacology study on B-cell depletion in cynomolgus monkeys (see non-clinical pharmacology studies section). In this study, male cynomolgus monkeys were administered a single dose of rituximab SC at $20 \mathrm{mg} / \mathrm{kg}$ [17]. The 
Table 3 Non-compartmental PK parameters for rituximab in female minipigs following single SC administration of rituximab with varying recombinant human hyaluronidase concentrations (mean \pm SD).

\begin{tabular}{|c|c|c|c|c|}
\hline \multirow[t]{2}{*}{ Parameter } & \multicolumn{4}{|c|}{ Dosing regimen ( $n=5 /$ dose group) } \\
\hline & $120 \mathrm{mg} / 0 \mathrm{U} / \mathrm{mL}$ rHuPH20 & $120 \mathrm{mg} / 2000 \mathrm{U} / \mathrm{mL}$ rHuPH 20 & $120 \mathrm{mg} / 6000 \mathrm{U} / \mathrm{mL}$ rHuPH20 & $240 \mathrm{mg} / 2000 \mathrm{U} / \mathrm{mL}$ rHuPH20 \\
\hline$C_{\max }(\mu \mathrm{g} / \mathrm{mL})$ & $82.6 \pm 15.8$ & $110 \pm 26.2$ & $110 \pm 33.3$ & $230 \pm 37.3$ \\
\hline$T_{\max }{ }^{a}(h)$ & 48 & 24 & 24 & 36 \\
\hline AUC $_{0-672 \mathrm{~h}}\left(\mathrm{~h}^{*} \mu \mathrm{g} / \mathrm{mL}\right)$ & $24100 \pm 10600$ & $32700 \pm 8060$ & $26300 \pm 12300$ & $52800 \pm 16200$ \\
\hline$F(\%)$ & $52.4 \pm 29.2$ & $70.7 \pm 28.1$ & $57.4 \pm 33.2$ & $57.8 \pm 25.7$ \\
\hline
\end{tabular}

${ }^{a}$ Median value. F: bioavailability; $T_{\max }$ : time to maximum serum concentration

Table 4 Treatment groups in non-clinical xenograft study in employing the Z138 human mantle cell lymphoma cell line in female SCID beige mice and rituximab serum trough concentrations after first and last rituximab administration.

\begin{tabular}{|c|c|c|c|c|}
\hline \multirow[t]{2}{*}{ Group } & \multirow[t]{2}{*}{ Dose $(\mathrm{mg} / \mathrm{kg})$} & \multirow[t]{2}{*}{ Administration route } & \multicolumn{2}{|c|}{ Rituximab serum trough concentration $(\mu \mathrm{g} / \mathrm{mL}$; [mean $\pm \mathrm{SD}]$} \\
\hline & & & After first administration & After third administration \\
\hline 1 (vehicle group) & NA & & & \\
\hline 2 & 3 & IV & $16.9 \pm 4.8$ & $30.0 \pm 14.2$ \\
\hline 3 & 10 & IV & $46.8 \pm 6.5$ & $70.3 \pm 18.6$ \\
\hline 4 & 30 & IV & $152.6 \pm 33.9$ & $224.3 \pm 43.3$ \\
\hline 5 & 5 & SC & $22.0 \pm 1.9$ & $33.6 \pm 8.6$ \\
\hline 6 & 16 & SC & $74.9 \pm 24.3$ & $115.9 \pm 37.1$ \\
\hline 7 & 50 & SC & $181.8 \pm 35.8$ & $246.7 \pm 82.7$ \\
\hline 8 (mice without tumor) & 10 & IV & $43.5 \pm 6.2$ & $124 \pm 18.1$ \\
\hline
\end{tabular}

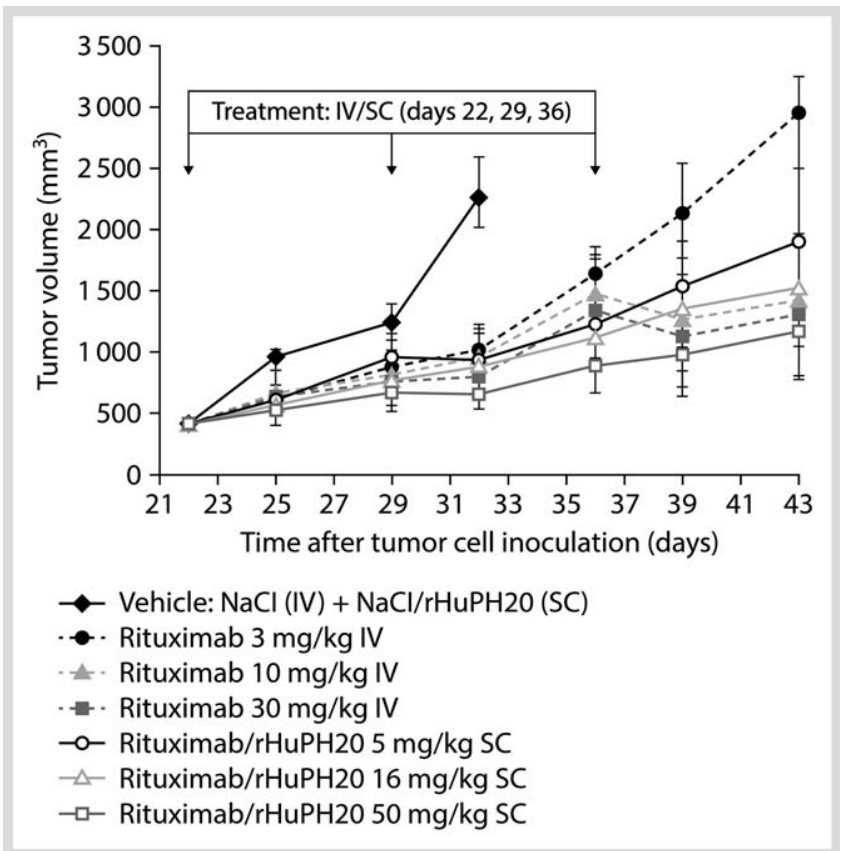

Fig. 2 Treatment effects in a non-clinical xenograft study employing the Z138 human mantle cell lymphoma cell line in female SCID beige mice.

rituximab SC formulation contained rHuPH20 at a nominal concentration of $6000 \mathrm{U} / \mathrm{mL}$. Maximum serum concentrations of $300 \pm 10.8 \mu \mathrm{g} / \mathrm{mL}$ rituximab (mean \pm SD) were reached by $24 \mathrm{~h}$ post dose. The average apparent clearance (clearance divided by bioavailability [CL/F]) after SC administration was $7.23 \mathrm{~mL} /$ day/ $\mathrm{kg}$. The SC bioavailability of rituximab was estimated to be close to complete, based on a comparison with available data after IV administration of rituximab to cynomolgus monkeys (data on file, F. Hoffmann-La Roche Ltd.).

\section{Non-Clinical Pharmacology Studies}

Non-clinical pharmacology studies have been conducted to support the hypothesis that comparable rituximab serum trough concentrations following SC and IV administration result in the same efficacy, regardless of the route of administration.

The pharmacodynamic effects of rituximab SC were compared with those of rituximab IV in a xenograft model employing the Z138 human mantle cell lymphoma cell line [18] in female SCID beige mice (data on file, F. Hoffmann-La Roche). Corresponding IV and SC dosages yielding a similar range of rituximab trough concentration levels were selected based on a PK study conducted in the same species, in which the rituximab SC bioavailability was estimated at $62 \%$ (data on file, F. Hoffmann-La Roche Ltd.). In the xenograft model study, mice received 3 treatments weekly on days 22, 29 and 36 following tumor cell inoculation at 3,10 or $30 \mathrm{mg} / \mathrm{kg}$ IV or at 5,16 or $50 \mathrm{mg} / \mathrm{kg}$ SC ( $\odot$ Table 4 ). An additional group without tumors was dosed at $10 \mathrm{mg} / \mathrm{kg} \mathrm{IV}$.

As illustrated in 0 Fig. 2, treatment with corresponding doses of $10 \mathrm{mg} / \mathrm{kg}$ IV and $16 \mathrm{mg} / \mathrm{kg} \mathrm{SC}$, or $30 \mathrm{mg} / \mathrm{kg}$ IV and $50 \mathrm{mg} / \mathrm{kg} \mathrm{SC}$, showed comparable anti-tumor activity. SC treatment at $5 \mathrm{mg} /$ $\mathrm{kg}$ was slightly more efficacious than IV treatment at $3 \mathrm{mg} / \mathrm{kg}$; however, this difference was not statistically significant. Serum levels of rituximab given at corresponding IV and SC doses were in the same range, with a maximum 2 -fold deviation ( $\bullet$ Table 4 ). It is of note that in the mid- and high-dose groups, the therapeutic effect may have reached saturation. Therefore, the comparison of IV and SC dosing regimens is best based on the results from the low-dose groups (i.e., the $3 \mathrm{mg} / \mathrm{kg}$ IV and $5 \mathrm{mg} / \mathrm{kg} \mathrm{SC}$ groups). The results of the xenograft tumor model study, particularly those from the low dose groups, suggest that when similar trough concentrations of rituximab are reached by SC and IV administration, similar efficacy is achieved in this xenograft model.

Mao et al. [17] studied the impact of the route of administration on B-cell depletion in cynomolgus monkeys. Animals were treated twice, one week apart, with either rituximab IV or ritux- 
imab SC $(2 \times 10 \mathrm{mg} / \mathrm{kg})$. The same dose level was used for both routes, as the preparatory PK study described earlier had indicated a close-to-complete SC bioavailability of rituximab in cynomolgus monkeys. Peripheral CD20-positive $(C D 20+)$ B-cell depletion was studied over a 2-month period. CD20 target coverage and the level of B-cell depletion in secondary lymphoid organs were measured at distal lymph nodes 9 days following the second dosing. While initial peak serum concentrations of rituximab were higher following IV administration, serum trough levels were comparable between dosing routes on days 2 , 7,9 and 14, i.e., 2 and 7 days after dosing on days 0 and 7, respectively. CD20 + B-cell depletion in blood was greater than 99\% compared with baseline for both rituximab SC and rituximab IV by day 9 (i.e., 2 days post-second dose). The duration of B-cell depletion was equally sustained 2 months after rituximab SC and IV dosing. Rituximab SC and IV both fully covered distal lymph node B-cell surface CD20, reducing the staining of free surface CD20 by $95 \%$ compared with baseline measurements. Administration via both modes also depleted lymph node B-cells to a similar extent 9 days after the second dose (SC: $57 \%$ vs. IV: $42 \%$. These results demonstrate that despite initial peak serum drug level differences, rituximab SC has a similar pharmacodynamic effect and durability compared with rituximab IV.

\section{Clinical Development Program}

$\nabla$

The early clinical development program to support the SC formulation in the FL indication was designed to identify a rituximab SC dose that would result in rituximab $C_{\text {trough }}$ serum levels at least as high as those achieved with standard doses of rituximab IV. Further clinical studies were designed to compare efficacy and safety with the 2 routes of administration.

\section{Rituximab Dose Selection for Clinical Dose-Finding Trial \\ $\nabla$}

To account for the comparatively high variability observed for the SC bioavailability of rituximab in animal models (see previous section on non-clinical PK studies), the initial clinical doses of rituximab SC were chosen to cover a bioavailability range between $60 \%$ and $100 \%$. Therefore, the selected rituximab starting dose for the SC formulation in the dose-finding trial was $375 \mathrm{mg} / \mathrm{m}^{2}$, assuming a SC bioavailability of $100 \%$. The second predefined dose was $625 \mathrm{mg} / \mathrm{m}^{2}$, assuming a SC bioavailability of $60 \%$. An additional SC dose of $800 \mathrm{mg} / \mathrm{m}^{2}$ was administered following interim PK analysis to account for a lower bioavailability of only $40 \%$. Generating PK data over a broad range of exposures was necessary for selecting a fixed SC dose for formal PK comparison with the approved IV regimen.

Readers are referred to previous publications for the supporting rationale and examples of fixed dosing of monoclonal antibodies $[7,19,20]$.

\section{Clinical Trials}

\section{$\nabla$}

A phase Ib study (SparkThera; NCT00930514/BP22333) was conducted in the maintenance setting in patients with previously treated or untreated FL (grade 1,2 or $3 \mathrm{a}$ ) who had responded to rituximab-based induction treatment. The maintenance setting was selected for dose finding because in this patient population, tumor load is lower and the risk of underdosing patients during dose finding was considered less critical than that associated with treating patients during induction treatment. Stage 1 of this study was designed to identify a fixed rituximab SC dose yielding rituximab $\mathrm{C}_{\text {trough }}$ levels comparable to those achieved after administration of rituximab IV $(375 \mathrm{mg} /$ $\mathrm{m}^{2}$ given every 2 or 3 months). As described previously [21], eligible patients who had received at least 1 dose of rituximab IV $\left(375 \mathrm{mg} / \mathrm{m}^{2}\right)$ in the maintenance setting were randomized to one of 4 rituximab maintenance treatment groups. Patients received a single dose of either rituximab IV $\left[375 \mathrm{mg} / \mathrm{m}^{2}(\mathrm{n}=16)\right]$ or rituximab SC $\left[375 \mathrm{mg} / \mathrm{m}^{2}(\mathrm{n}=34), 625 \mathrm{mg} / \mathrm{m}^{2} \quad(\mathrm{n}=34)\right.$, or $\left.800 \mathrm{mg} / \mathrm{m}^{2}(\mathrm{n}=40)\right]$. Following this single dose, all patients continued receiving maintenance doses of rituximab IV $(375 \mathrm{mg} /$ $\mathrm{m}^{2}$ ) every 2 or 3 months for a further year. At the end of this year, patients who had already received a single dose of rituximab SC had the option to receive rituximab SC at the final fixed dose $(1400 \mathrm{mg})$ for the remaining year of the 2-year maintenance period, or continue with rituximab IV ( $\odot$ Fig. 3a). The PK data generated in stage 1 were used to select the dose of rituximab SC for stage 2 of the study [22].

Stage 2 of SparkThera was designed to demonstrate serum $\mathrm{C}_{\text {trough }}$ noninferiority of the selected rituximab SC dose compared with that of the established rituximab IV dose ( $\bullet$ Fig. $3 \mathbf{b}$ ). In total, 154 patients were randomized 1:1 to receive rituximab SC $(1400 \mathrm{mg})$ or rituximab IV $\left(375 \mathrm{mg} / \mathrm{m}^{2}\right)$ for their remaining maintenance cycles. In line with the results from the minipig and mice studies, the SC bioavailability was estimated at $65 \%$ in stage 1 and $69 \%$ in stage 2 of the study. The study met its primary endpoint; specifically, the minimum rituximab concentration ratio for SC vs. IV was 1.24 when rituximab was given once every 2 months and 1.12 when rituximab was given once every 3 months [22] ( $\bullet$ Table 5). The $1400 \mathrm{mg}$ rituximab SC dose was therefore selected for study in the SABRINA phase 3 trial [23]. SABRINA (NCT01200758/BO22334) is a two-stage, international, phase III trial designed to investigate the PK, efficacy and safety of SC vs. IV administration of rituximab in patients with FL (grade 1, 2 or 3a) receiving induction and maintenance therapy ( $\bullet$ Fig. 3c). In stage 1 of the trial, previously untreated patients were randomized to receive rituximab IV $375 \mathrm{mg} / \mathrm{m}^{2}$ or a fixed dose of $1400 \mathrm{mg}$ of rituximab SC, both given in combination with either CHOP (cyclophosphamide, doxorubicin, vincristine, and prednisone) or CVP (cyclophosphamide, vincristine and prednisone) chemotherapy. Patients who achieved a complete or partial response after 8 treatment cycles continued rituximab maintenance therapy as per their initial randomization with either SC or IV administration. The primary endpoint for stage 1 was to estimate the ratio of rituximab serum concentrations $\left(\mathrm{C}_{\text {trough, sc }}: \mathrm{C}_{\text {trough, IV }}\right)$ at cycle 7 during induction treatment; the ratio was 1.62 (90\% CI: $1.36-1.94)$, which met the limit for noninferiority of $\mathrm{C}_{\text {trough, } \mathrm{SC}}($ ratio $>0.80)$ [23]. In addition, exploratory efficacy analyses demonstrated similar overall response rates (ORR; 84.4\% IV and 90.5\% SC) and complete response (CR) rates (29.7\% IV and $46 \%$ SC). In the ongoing stage 2 portion of the study, efficacy is the primary endpoint and additional patients are being randomized to receive either SC or IV administration of rituximab. Data obtained from this trial were presented at the $54^{\text {th }}$ American Society of Hematology Annual Meeting in Atlanta [23] and have been submitted in a marketing application to regulatory authorities in the European Union. 


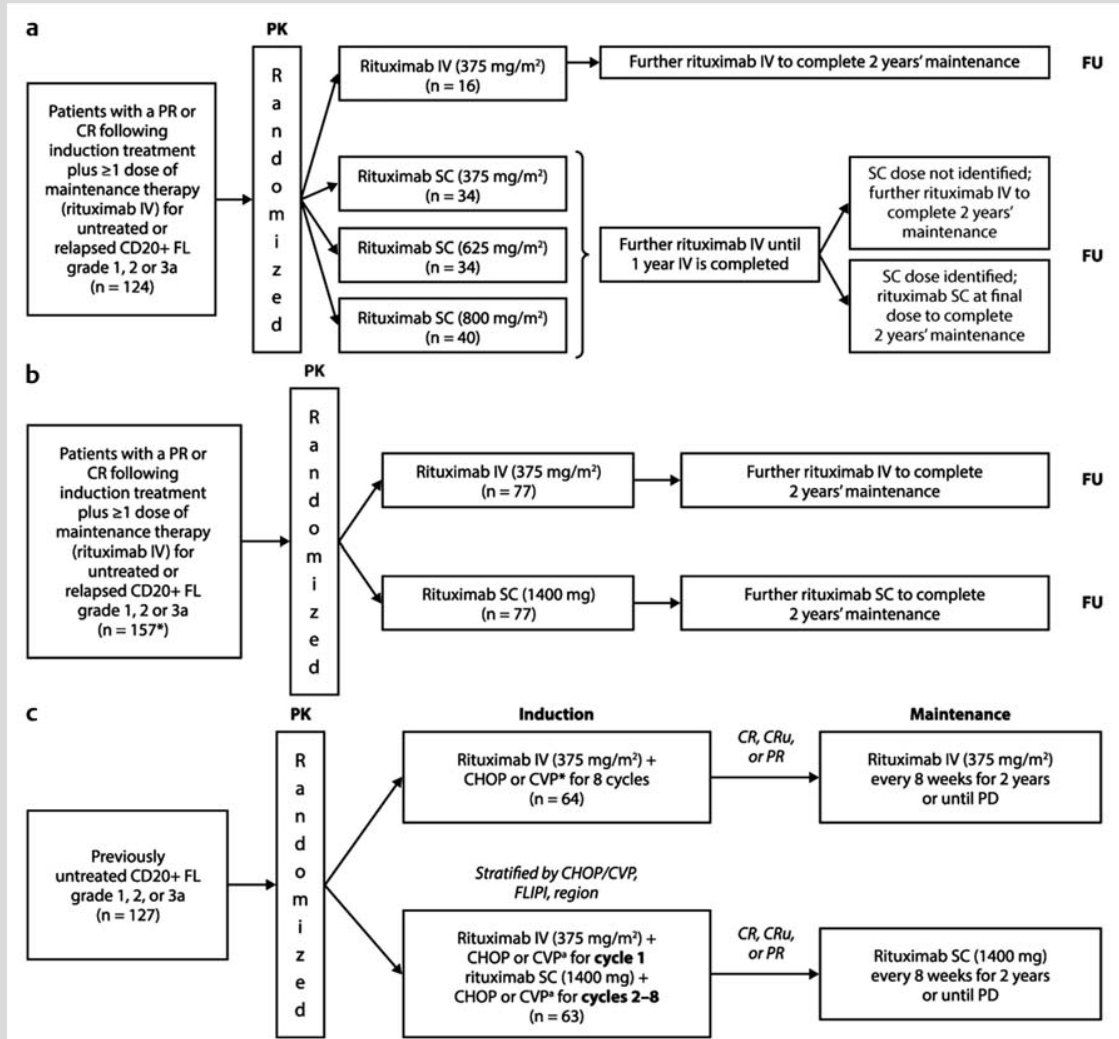

Fig. 3 Clinical trial schemas. a SparkThera phase Ib clinical trial: stage 1, dose finding. b SparkThera phase Ib clinical trial: stage 2 , dose confirmation. c SABRINA phase III trial. ${ }^{a} 3$ patients were withdrawn prior to treatment. ${ }^{\mathrm{b}} \mathrm{CHOP}$ : administered as cyclophosphamide $\left(750 \mathrm{mg} / \mathrm{m}^{2} \mathrm{IV}\right.$ on day 1$)$, doxorubicin $\left(50 \mathrm{mg} / \mathrm{m}^{2} \mathrm{IV}\right.$ on day 1$)$, vincristine $\left[1.4 \mathrm{mg} / \mathrm{m}^{2} \mathrm{IV}\right.$ on day 1 ( $2 \mathrm{mg}$ maximum)], and prednisone (100 $\mathrm{mg}$ oral or IV on days $1-5)$ in every cycle. CVP: administered as cyclophosphamide $\left(750 \mathrm{mg} / \mathrm{m}^{2}\right.$ IV on day 1$)$, vincristine $[1.4 \mathrm{mg} /$ $\mathrm{m}^{2} \mathrm{IV}$ on day 1 ( $2 \mathrm{mg}$ maximum)], and prednisone $\left(40 \mathrm{mg} / \mathrm{m}^{2}\right.$ oral or IV on days $\left.1-5\right)$ in every cycle.

CRu: unconfirmed complete response; CVP: cyclophosphamide, vincristine and prednisone; FLIPI: Follicular Lymphoma International Prognostic Index; FU, follow-up.

Table 5 Expected $C_{\text {trough }}$ levels stage 2 SparkThera trial.

\begin{tabular}{|c|c|c|c|c|}
\hline Dosing regimen & Parameter & SC $1400 \mathrm{mg}(n=77)$ & IV $375 \mathrm{mg} / \mathrm{m}^{2}(\mathrm{n}=76)$ & Geometric mean ratio $C_{\text {trough, sc }}: C_{\text {trough, Iv }}[90 \% \mathrm{Cl}]$ \\
\hline $\mathrm{q} 2 \mathrm{~m}$ & Geometric mean $\pm S D$ & $32.2 \pm 29.1$ & $25.9 \pm 16.3$ & $1.24[1.02-1.51]$ \\
\hline$q 3 m$ & Geometric mean $\pm S D$ & $12.1 \pm 16.5$ & $10.9 \pm 9.9$ & $1.12[0.86-1.45]$ \\
\hline
\end{tabular}

\section{Summary and Outlook}

This review describes the early development of a novel SC formulation of rituximab. This formulation is expected to offer an attractive alternative to the more invasive and lengthier IV administration. Similar to the trastuzumab example [7], rituximab SC maintains the same administration frequency as the corresponding IV formulation, but is given as a fixed dose instead of the BSA-adjusted dose used for rituximab IV. ORR and CR data from stage 1 of the ongoing SABRINA trial support the validity of the basic bridging assumption used to calculate the SC dose, and demonstrate that rituximab SC serum trough concentrations that are at least as high as those achieved with IV dosing result in at least the same degree of clinical efficacy.

Using the SC formulation of rituximab may shorten treatment time significantly, by enabling administration in less than 10 min. Furthermore, the ready-to-use SC formulation has the potential to significantly reduce both medicine preparation time and hospital staff time per administration. Therefore, use of the rituximab SC formulation instead of the IV formulation is also expected to reduce both pharmacy time and the burden placed on hospital resources, as well as having the potential to provide improved patient convenience.

\section{Acknowledgements}

Support for third-party editorial assistance for this manuscript was provided by F. Hoffmann-La Roche Ltd.

\section{Conflict of Interest}

Drs. B. Bittner, W. Richter, F. Hourcade-Potelleret, F. Herting and J. Schmidt are former or current employees of F. Hoffmann-La Roche Ltd.

\section{References}

1 Maloney DG, Grillo-López AJ, White CA et al. IDEC-C2B8 (rituximab) anti-CD20 monoclonal antibody therapy in patients with relapsed low-grade non-Hodgkin's lymphoma. Blood 1997; 90: 2188-2195

2 Plosker GL, Figgitt DP. Rituximab: a review of its use in non-Hodgkin's lymphoma and chronic lymphocytic leukaemia. Drugs 2003; 63: 803-843

3 Coiffier B, Lepage E, Brière J et al. CHOP chemotherapy plus rituximab compared with CHOP alone in elderly patients with diffuse large-Bcell lymphoma. N Engl J Med 2002; 346: 235-242

4 Shivakumar SP, Anderson DR, Couban S. Catheter-associated thrombosis in patients with malignancy. J Clin Oncol 2009; 27: 4858-4864

5 Barber ND, Hoffmeyer UK. Comparison of the cost-effectiveness of administering heparin subcutaneously or intravenously for the treatment of deep vein thrombosis. Ann R Coll Surg Engl 1993; 75: 430-433 
6 Pivot X, Gligorov J, Muller $V$ et al. Patient preference for subcutaneous versus intravenous adjuvant trastuzumab: results of the PREFHER study. The Breast 2013; 22 (Suppl 1): S87 (Abstract 207)

7 Bittner B, Richter WF, Hourcade-Potelleret $F$ et al. Development of a subcutaneous formulation for trastuzumab - nonclinical and clinical bridging approach to the approved intravenous dosing regimen. Arzneimittelforschung 2012; 62: 401-409

8 Wynne C, Harvey V, Schwabe $C$ et al. Comparison of subcutaneous and intravenous administration of trastuzumab: a phase I/ Ib trial in healthy male volunteers and patients with HER2-positive breast cancer. J Clin Pharmacol 2012, [epub ahead of print] doi:10.1177/0091270012436560

9 Ismael G, Hegg R, Muehlbauer S et al. Subcutaneous versus intravenous administration of (neo)adjuvant trastuzumab in patients with HER2positive, clinical stage I-III breast cancer (HannaH study): a phase 3, open-label, multicenter, randomized trial. Lancet Oncol 2012; 13 : 869-878

10 MabThera $^{\mathrm{TM}}$ (rituximab) summary of product characteristics 2009; Available from http://www.mabthera.com/portal/mabthera/safety_ information

11 Dani B, Platz R, Tzannis ST. High concentration formulation feasibility of human immunoglobulin $G$ for subcutaneous administration. J Pharm Sci 2007; 96: 1504-1517

12 Shire SJ, Shahrokh Z, Liu J. Challenges in the development of high protein concentration formulations. J Pharm Sci 2004; 93: 1390-1402

13 Frost GI. Recombinant human hyaluronidase (rHuPH20): An enabling platform for subcutaneous drug and fluid administration. Expert Opin Drug Deliv 2007; 4: 427-440

14 By Adler M, Mahler $H-C$, Stauch OB. Highly concentrated pharmaceutical formulations. Patent application submitted in the European Union (Patent application number: 20110076273). Available at: http:// www.faqs.org/patents/app/20110076273

15 Zheng Y, Tesar DB, Benincosa L et al. Minipig as a potential translatable model for monoclonal antibody pharmacokinetics after intravenous and subcutaneous administration. MAbs 2012; 4: 243-255
16 Yocum RC, Vaughn DE, Mongiovi DJ et al. Clinical trial of recombinant human PH20 hyaluronidase's effect on subcutaneous absorption and pharmacokinetics of a large protein molecule therapeutic. Proceedings of the $34^{\text {th }}$ Annual Meeting and Exposition of the Controlled Release Society 2007, held 7-11 July 2007, Long Beach, California

17 Mao CP, Brovarney MR, Dabbagh K et al. Subcutaneous versus intravenous administration of rituximab: Pharmacokinetics, CD20 target coverage and B-cell depletion in Cynomolgus monkeys. PlosOne [accepted]

18 Tucker CA, Bebb G, Klasa RJ et al. Four human t(11;14)(q13;q32)-containing cell lines having classic and variant features of Mantle Cell Lymphoma. Leuk Res 2006; 30: 449-457

19 Ng CM, Lum BL, Gimenez V et al. Rationale for fixed dosing of pertuzumab in cancer patients based on population pharmacokinetic analysis. Pharm Res 2006; 23: 1275-1284

20 Wang DD, Zhang S, Zhao H et al. Fixed dosing versus body size-based dosing of monoclonal antibodies in adult clinical trials. J Clin Pharmacol 2009; 49: 1012-1024

21 Salar A, Bouabdallah R, McIntyre C et al. A two-stage phase Ib study to investigate the pharmacokinetics, safety and tolerability of subcutaneous rituximab in follicular lymphoma as part of maintenance treatment. Blood 2010; 116: (Abstract 2858)

22 Salar A, Avivi I, Larouche JF et al. Final results of the BP22333 study demonstrate non-inferior pharmacokinetics and safety of subcutaneous administration of rituximab compared with intravenous administration as maintenance therapy in patients with follicular lymphoma. Blood 2012; 120: (Abstract 1641)

23 Davies A, Merli F, Mihaljevic B et al. Pharmacokinetics (PK), safety and overall response rate (ORR) achieved with subcutaneous (SC) administration of rituximab in combination with chemotherapy were comparable to those achieved with intravenous (IV) administration in patients (pts) with follicular lymphoma (FL) in the first-line setting: stage 1 results of the phase III SABRINA study (BO22334). Blood 2012; 120: (Abstract 1629) 